This item was submitted to Loughborough's Research Repository by the author.

Items in Figshare are protected by copyright, with all rights reserved, unless otherwise indicated.

\title{
Model predictive control of low Earth-orbiting satellites using magnetic
} actuation

PLEASE CITE THE PUBLISHED VERSION

PUBLISHER

(C) IMechE / Professional Engineering Publishing

LICENCE

CC BY-NC-ND 4.0

REPOSITORY RECORD

Wood, Mark, and Wen-Hua Chen. 2008. "Model Predictive Control of Low Earth-orbiting Satellites Using Magnetic Actuation". figshare. https://hdl.handle.net/2134/3755. 
This item was submitted to Loughborough's Institutional Repository (https://dspace.lboro.ac.uk/) by the author and is made available under the following Creative Commons Licence conditions.

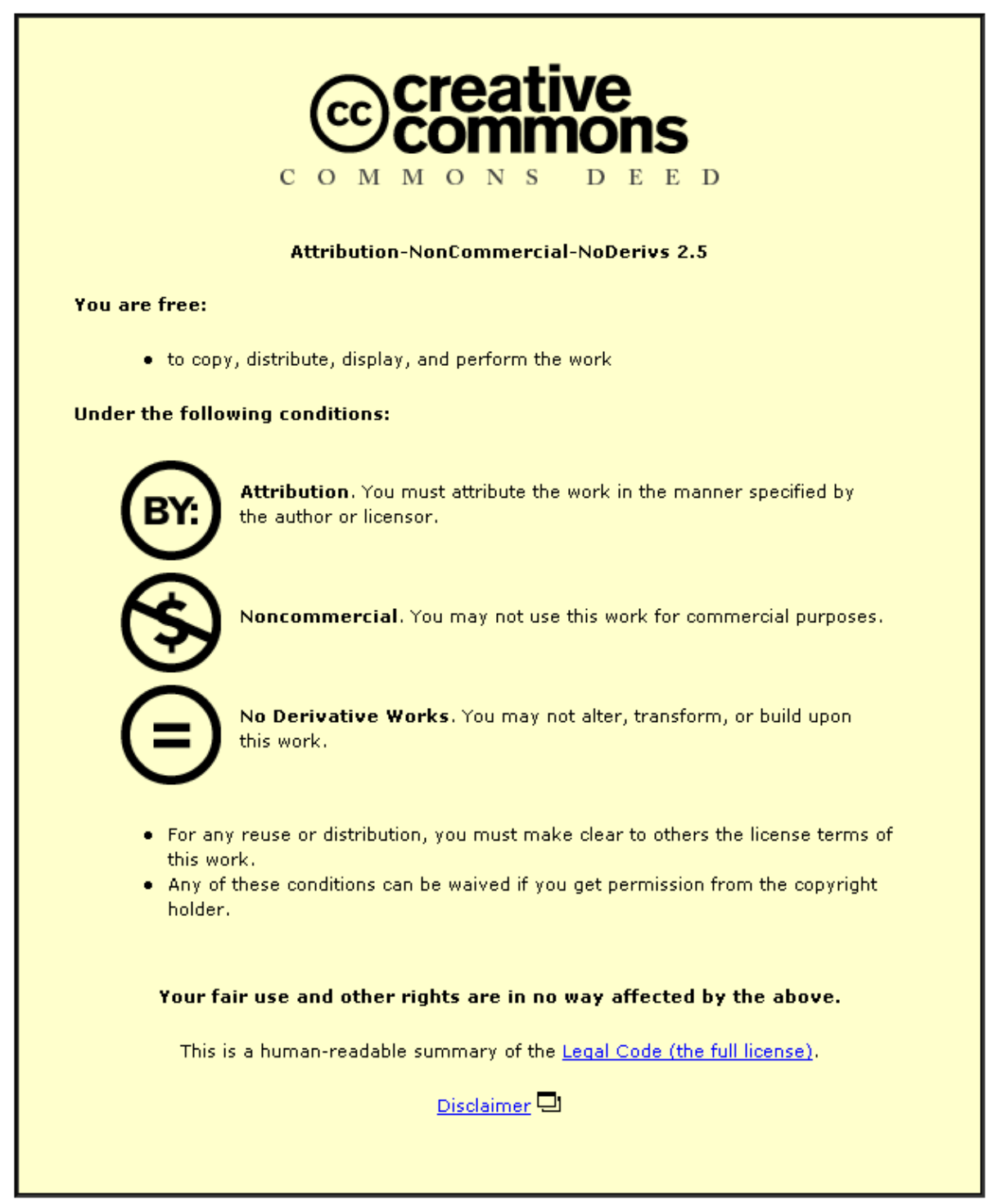

For the full text of this licence, please go to: http://creativecommons.org/licenses/by-nc-nd/2.5/ 


\title{
Model predictive control of low Earth-orbiting satellites using magnetic actuation
}

\author{
M Wood and W H Chen* \\ Aeronautical and Automotive Engineering, Loughborough University, Loughborough, UK
}

The manuscript was received on 15 October 2007 and was accepted after revision for publication on 14 April 2008.

DOI: 10.1243/09596518JSCE505

\begin{abstract}
This paper presents a model predictive control approach for regulating the attitude of magnetically actuated satellites. Unlike other contributions in this area, a predictive control approach is developed which guarantees closed-loop stability of satellite configurations with unstable open-loop pitch dynamics. With the pitch axis being unstable, two magnetic dipoles are used exclusively for regulation of this axis. This allows the dynamics to be treated as a linear time-invariant system, and a simple proportional-derivative (PD) scheme is implemented. A model predictive controller is designed to regulate the lateral dynamics, with a Lyapunov function derived to guarantee asymptotic stability of the closed-loop system. The regulation of the lateral dynamics is achieved with a singe dipole moment, with a novel reformulation of the lateral dynamics also providing an explicit link between the two controllers. Simulations demonstrate the effectiveness and stability of the proposed algorithm when applied to the European Space Agency's GOCE satellite.
\end{abstract}

Keywords: model predictive control, magnetic attitude control, stability constrained predictive control

\section{INTRODUCTION}

The area of spacecraft magnetic attitude control is one that has attracted much recent attention in research literature. Use of magnetic dipoles to control the attitude of a spacecraft offers a lightweight, smooth, and cost-effective method of control. Although this is the case, the torque generated through use of magnetic dipoles is constrained to lie in the plane orthogonal to the local magnetic field vector, with one axis being instantaneously underactuated. If the satellite is on an inclined orbit, suitable variation of the magnetic field allows controllability in the long term, but presents a significant challenge from a control perspective.

Several different approaches to the magnetic attitude control problem have been proposed by different authors, varying in type and complexity [116]. Perhaps the most basic approach suggested is use of a simple proportional-derivative (PD) con-

*Corresponding author: Aeronautical and Automotive Engineering, Loughborough University, Ashby Road, Loughborough LE11 3TU, UK. email: W.Chen@lboro.ac.uk troller, with the control signal being calculated as if full controllability were available. This 'ideal' control signal is then projected onto the controllable plane. This is an approach adopted in reference [1] and more recently in reference [2]. The use of a PD-based controller is also considered in reference [3], although such a basic control law is not seen to be suitable, and the controller is modified to vary as a function of the magnetic field to improve roll axis performance.

Although PD control is a simple approach to the problem, it cannot always give the desired performance. As a result, several authors try to exploit the pseudo-periodic nature of the Earth's magnetic field to use periodic linear quadratic control theory. Such an approach is presented in reference [4], with the resulting control law depending on the solution of the time-varying Riccati equation. Under certain assumptions, the solution to the Riccati equation tends to a constant value and hence the final controller simplifies to a state feedback controller, with the only time variance of the problem appearing from the magnetic field vector (which can easily be measured by onboard magnetometers). A 
periodic quadratic controller similar to this approach is presented in reference [5], but this is then extended to consider optimal periodic disturbance rejection.

In addition to optimal periodic control, researchers consider other approaches to the problem. A time-varying PD-like control approach consisting of full state feedback control is proposed in references [6] and [7]. Almost global stability is demonstrated, while also taking into account some practical aspects such as actuator failure and control without rate feedback. A sliding mode controller as well as an energy-based method are suggested as possible solutions to the attitude control problem in reference [8].

Model predictive control (MPC) is potentially a suitable candidate for dealing with the magnetic attitude problem, with its ability to use the measured magnetic field within the control law and to easily include constraints within the optimization scheme. In reference [9] a novel approach to the attitude control problem is presented by considering the system as time invariant, and introducing the time variance through an appropriate set of constraints. An explicit model predictive control scheme is presented in reference [10], with an aim to reducing the computational burden imposed as much as possible. This approach suitably removes large initial Euler angles and, when compared to other nonlinear controllers, presents onboard energy savings of up to 50 per cent. Although both of these approaches demonstrate good overall performance, the issue of stability is not yet answered. Floquet analysis is suggested to verify stability [9], although this is an after-the-fact check and stability cannot be guaranteed a priori.

On the stability of magnetic attitude regulation the main recent contributions come from references [4], [6], and [11], and all derive controllers based on open-loop stable satellite configurations. These provide some very interesting contributions to the research literature but are inapplicable for the regulation of satellites about an unstable equilibrium. The Gravity Field and Steady State Ocean Circulation Explorer (GOCE) satellite discussed in this paper has mission requirements such that the inertia distribution leads to unstable pitch dynamics. Within the literature the only notable contributions presenting stability analysis that can be applied to satellites with unstable pitch dynamics are references [5] and [15]. Both of these approaches rely on the assumption that the magnetic field is periodic at the orbital frequency, which is a common but strictly incorrect assumption to make (a point also noted in references [5] and [6]).

The main aim of the current paper is to provide the first model predictive approach to magnetic attitude control for which stability can be guaranteed a priori. In addition the analysis is designed specifically to guarantee stability even for a satellite with an open-loop unstable pitch configuration. Finally the analysis is carried out without relying on the frequently adopted assumption that the Earth's magnetic field is periodic at the orbital frequency.

This paper is structured as follows. Section 2 introduces the benchmark satellite and the dynamic equations. With the attitude regulation problem being considered, a linear model is sufficient to fully describe the attitude dynamics. Section 3 briefly introduces the MPC approach, while section 4 considers a novel reformulation of the attitude dynamics. This allows the pitch controller to be considered as a linear time-variant (LTI) problem while also providing an explicit equation to determine the magnetic dipole vector.

A stability enforced model predictive controller consisting of full state feedback is proposed for regulation of the lateral dynamics. A suitable stability constraint is derived by consideration of a velocity feedback controller, and asymptotic stability about all three satellite axes is demonstrated. Section 5 considers performance of the proposed controller design under initial conditions and also to a series of environmental disturbances.

\section{SPACECRAFT DYNAMICS UNDER MAGNETIC CONTROL}

Set for launch in 2008, the GOCE satellite is part of the European Space Agency's (ESA) living planet programme. The main aim of the satellite is to measure the Earth's gravity field gradient and hence the control aim is to cancel all non-gravitational angular accelerations acting upon GOCE. The selected orbit is a sun-synchronous, near-polar orbit of $96.5^{\circ}$ inclination, at an altitude of $270 \mathrm{~km}$, with an orbital period of approximately $5400 \mathrm{~s}$. The inertia distribution is summarized in Table 1, leading to an

Table 1 Satellite inertia distribution

\begin{tabular}{lc}
\hline Satellite principal axis & Inertia $\left(\mathrm{kg} \mathrm{m}^{2}\right)$ \\
\hline Roll $\left(I_{x x}\right)$ & 152.2 \\
Pitch $\left(I_{y y}\right)$ & 2690.8 \\
Yaw $\left(I_{z z}\right)$ & 2652.6 \\
\hline
\end{tabular}


unstable pitch and neutrally stable lateral configuration. For attitude regulation GOCE is equipped with three mutually perpendicular magnetic dipole rods each with $400 \mathrm{Am}^{2}$ saturation limit. The performance requirements of the attitude controller can be found in Table 2, while the interested reader may consult reference [3] for further information on the GOCE mission.

Although the true spacecraft dynamics are fully described by a series of non-linear differential equations, under certain assumptions these equations can be linearized with minimal loss of accuracy. If linearization is carried out about the equilibrium nadir-pointing attitude - assuming a circular orbit, small Euler angles, and deviation of body rates from nominal values - the following linearized model can be produced [4]. Once a satellite has acquired an Earth pointing attitude on orbit (i.e. once the initial high pointing angle and angular rates have been removed through non-linear control), the satellite dynamics are approximated well by such a linear model

$$
\dot{\boldsymbol{x}}(t)=\mathbf{A} \boldsymbol{x}(t)+\left[\begin{array}{l}
0_{3,3} \\
\mathbf{I}^{-1}
\end{array}\right] \boldsymbol{T}(t)
$$

where

$$
\begin{aligned}
& \mathbf{A}= \\
& {\left[\begin{array}{cccccc}
0 & 0 & 0 & 1 & 0 & 0 \\
0 & 0 & 0 & 0 & 1 & 0 \\
0 & 0 & 0 & 0 & 0 & 1 \\
-4 \omega_{0}^{2} \sigma_{1} & 0 & 0 & 0 & 0 & \omega_{0}\left(1-\sigma_{1}\right) \\
0 & 3 \omega_{0}^{2} \sigma_{2} & 0 & 0 & 0 & 0 \\
0 & 0 & \omega_{0}^{2} \sigma_{3} & -\omega_{0}\left(1+\sigma_{3}\right) & 0 & 0
\end{array}\right]} \\
& \boldsymbol{x}(t)=\left[\begin{array}{llllll}
\varphi & \theta & \psi & \omega_{x} & \omega_{y} & \omega_{z}
\end{array}\right]^{T}
\end{aligned}
$$

where $\phi, \theta, \psi$ are the spacecraft pointing angles about roll, pitch, and yaw axes respectively; $\omega_{x}, \omega_{y}$, $\omega_{z}$ are the spacecraft angular rates about roll, pitch, and yaw axes respectively; $\omega_{0}$ is the orbital rate; $\mathbf{I}$ is the inertia matrix; $\boldsymbol{T}$ is the control torque (with components $\left.T_{x}, T_{y}, T_{z}\right)$; and $\sigma_{i}=\left(I_{j}-I_{k}\right) / I_{i}$ for the index sets $(1,2,3),(2,3,1)$, and $(3,1,2)$.
Note that the co-ordinate system used throughout this paper defines the spacecraft orientation relative to a local-level co-ordinate system. The local-level system has the $+z$ axis pointing towards the nadir, the $y$ axis perpendicular to the orbital plane (defined by position and velocity vector), and the $x$ axis defined by the right-hand rule.

When considering magnetically controlled spacecraft, torque-rods generate control torques through interaction with the Earth's own magnetic field. This torque is perpendicular to the Earth's magnetic field vector and is described below in equation (2)

$$
\boldsymbol{T}=\boldsymbol{M} \times \boldsymbol{B}_{\mathrm{m}}
$$

where $\boldsymbol{M}$ is the vector of magnetic dipole moments (with components $M_{x}, M_{y}, M_{z}$ ) and $\boldsymbol{B}_{\mathrm{m}}$ is the Earth's magnetic field vector (with components $B_{\mathrm{m}_{x}}$, $\left.B_{\mathrm{m}_{y}}, B_{\mathrm{m}_{z}}\right)$.

\section{MODEL PREDICTIVE CONTROL}

\subsection{The MPC approach}

MPC is an advanced control strategy based largely around an internal model of the plant under control. This model is used to predict the expected future behaviour of the plant, with the resulting information being used to determine the 'optimal' control input to be applied. A typical MPC strategy adopts the following basic procedure.

1. An internal model is used to predict the future satellite behaviour over a finite future time period known as the prediction horizon.

2. This predicted behaviour can be compared to a desired reference trajectory, with an error vector being generated.

3. The optimum control sequence is defined by minimizing the predicted error over the prediction horizon.

4. The first input in the 'optimal' control sequence is applied to the plant, with the remaining sequence being discarded and the whole process then repeated at the next sampling interval.

Table 2 GOCE performance requirements

\begin{tabular}{llll}
\hline Axis & Max. pointing angle $(\mathrm{deg})$ & Max. angular rate $(\mathrm{rad} / \mathrm{s})$ & Max. angular acc. $\left(\mathrm{rad} / \mathrm{s}^{2}\right)$ \\
\hline Roll & 8 & $2 \mathrm{e}-4$ & $1.8 \mathrm{e}-6$ \\
Pitch & 3 & $3 \mathrm{e}-5$ & $0.9 \mathrm{e}-6$ \\
Yaw & 8 & $2 \mathrm{e}-4$ & $0.9 \mathrm{e}-6$ \\
\hline
\end{tabular}




\subsection{Control problem formulation}

Consider a discrete linear time-varying system of the following form

$$
\boldsymbol{x}(\boldsymbol{k}+1)=\boldsymbol{\Phi} \boldsymbol{x}(\boldsymbol{k})+\boldsymbol{\Gamma}(\boldsymbol{k}) \boldsymbol{u}(\boldsymbol{k})
$$

Equation (3) can be iterated over a finite future period to form predictions of the future state vector in terms of the current state and a future control sequence

$$
\begin{aligned}
\boldsymbol{X} & =\left[\begin{array}{c}
\boldsymbol{\Phi} \\
\boldsymbol{\Phi}^{2} \\
\boldsymbol{\Phi}^{3} \\
\vdots \\
\boldsymbol{\Phi}^{N}
\end{array}\right] \boldsymbol{x}(\boldsymbol{k}) \\
& +\left[\begin{array}{cccc}
\boldsymbol{\Gamma}(\boldsymbol{k}) & & & \\
\boldsymbol{\Phi} \boldsymbol{\Gamma}(\boldsymbol{k}) & \boldsymbol{\Gamma}(\boldsymbol{k}+1) & 0 & 0 \\
\boldsymbol{\Phi}^{2} \boldsymbol{\Gamma}(\boldsymbol{k}) & \boldsymbol{\Phi} \boldsymbol{\Gamma}(\boldsymbol{k}+1) & \ddots & 0 \\
\vdots & \vdots & \ddots & \vdots \\
\boldsymbol{\Phi}^{N-1} \boldsymbol{\Gamma}(\boldsymbol{k}) & \boldsymbol{\Phi}^{N-2} \boldsymbol{\Gamma}(\boldsymbol{k}+1) & \cdots & \boldsymbol{\Gamma}(\boldsymbol{k}+\boldsymbol{N}-1)
\end{array}\right] U
\end{aligned}
$$

where

$$
\begin{aligned}
X & =\left[\begin{array}{llll}
\hat{\boldsymbol{x}}(\boldsymbol{k}+1) & \hat{\boldsymbol{x}}(\boldsymbol{k}+2) & \cdots & \hat{\boldsymbol{x}}(\boldsymbol{k}+\boldsymbol{N})
\end{array}\right]^{T} \\
U & =\left[\begin{array}{llll}
\hat{\boldsymbol{u}}(\boldsymbol{k}) & \hat{\boldsymbol{u}}(\boldsymbol{k}+1) & \cdots & \hat{\boldsymbol{u}}(\boldsymbol{k}+\boldsymbol{N}-1)
\end{array}\right]^{T}
\end{aligned}
$$

$\boldsymbol{X}$ is the predicted future state vector, $\boldsymbol{U}$ is the future predicted control sequence, and $\boldsymbol{N}$ is the prediction horizon.

The predictions carried out in equation (4) can be used to determine an optimal control sequence over the prediction horizon to minimize a defined performance index. The performance index to be minimized typically takes the quadratic form shown in equation (5)

$$
\begin{aligned}
\boldsymbol{J}(\boldsymbol{k})= & \sum_{i=\mathbf{1}}^{N} \hat{\boldsymbol{x}}(\boldsymbol{k}+i)^{T} \mathbf{Q} \hat{\boldsymbol{x}}(\boldsymbol{k}+i)+\hat{\boldsymbol{u}}(\boldsymbol{k}+i-1)^{T} \mathbf{R} \hat{\boldsymbol{u}}(\boldsymbol{k}+i-1) \\
& +\hat{\boldsymbol{x}}(\boldsymbol{k}+N)^{T} \mathbf{Q}_{\mathbf{T}} \hat{\boldsymbol{x}}(\boldsymbol{k}+N)
\end{aligned}
$$

where $\mathbf{Q}>0$ is the state penalty weighting, $\mathbf{R}>0$ is the control weighting matrix, and $\mathbf{Q}_{\mathbf{T}}>0$ is the terminal penalty providing an additional weighting on the last state in the prediction horizon.

\subsection{Stability enforced MPC}

Stability of model predictive control is generally achieved through use of a carefully chosen terminal penalty (see reference [17]) or an explicit constraint within the optimization process (see reference [18]). The second method is relevant to this paper and is briefly described. According to reference [18] if the optimization problem in (5) is solved subject to the constraint $\boldsymbol{x}(\boldsymbol{k}+1)^{\boldsymbol{T}} \mathbf{P} \boldsymbol{x}(\boldsymbol{k}+1) \leqslant \boldsymbol{x}(\boldsymbol{k})^{\mathrm{T}} \mathbf{P} \boldsymbol{x}(\boldsymbol{k})-\delta(k)$, asymptotic stability of the closed-loop system is guaranteed. The stability argument proceeds as follows.

1. A discrete-time Lyapunov function is defined such that at time $k, V(k)=\boldsymbol{x}(\boldsymbol{k})^{\mathbf{T}} \mathbf{P} \boldsymbol{x}(\boldsymbol{k})$.

2. At time $k+1$ the Lyapunov function becomes $V(k+1)=\boldsymbol{x}(\boldsymbol{k}+1)^{\boldsymbol{T}} \mathbf{P} \boldsymbol{x}(\boldsymbol{k}+1)$.

3. The change of Lyapunov function is calculated as $V(k+1)-V(k)=\boldsymbol{x}(\boldsymbol{k}+1)^{\boldsymbol{T}} \mathbf{P} \boldsymbol{x}(\boldsymbol{k}+1)-\boldsymbol{x}(\boldsymbol{k})^{\boldsymbol{T}} \mathbf{P} \boldsymbol{x}(\boldsymbol{k})$.

4. Substitution of the stability constraint leads to $V(k+1)-V(k) \leqslant-\delta(k)$

5. Providing $\delta(k)>0$ the Lyapunov function decreases at each time step and asymptotic stability is guaranteed.

This approach provides a simple and intuitive method of guaranteeing stability; however, the analysis does make the assumption that the optimization problem in equation (5) remains feasible under the stability constraint. In order to implement this approach and ensure the stability argument remains valid, the $\mathbf{P}$ matrix must be carefully chosen to ensure a feasible control solution exists. This is a non-trivial problem for time-varying systems such as the magnetic attitude control problem considered in this paper. A systematic approach to tackle this difficulty will be proposed in section 4.3 .

\section{CONTROLLER DESIGN}

\subsection{Pitch control}

With the open-loop pitch dynamics unstable, the initial concern is to stabilize this axis. Owing to the nature of the Earth's magnetic field this is always possible. Figure 1 shows the variation of the Earth's magnetic field in spacecraft co-ordinates. A dipole model assuming no Earth rotation and no orbit precession is used (see reference [4]), but this is adequate to illustrate the controllability about the pitch axis.

From equation (2) the pitch torque can be calculated as $T_{y}=-B_{\mathrm{m}_{z}} M_{x}+B_{\mathrm{m}_{x}} M_{z}$. It is clear from Fig. 1 that the $X$ and $Z$ magnetic field components 


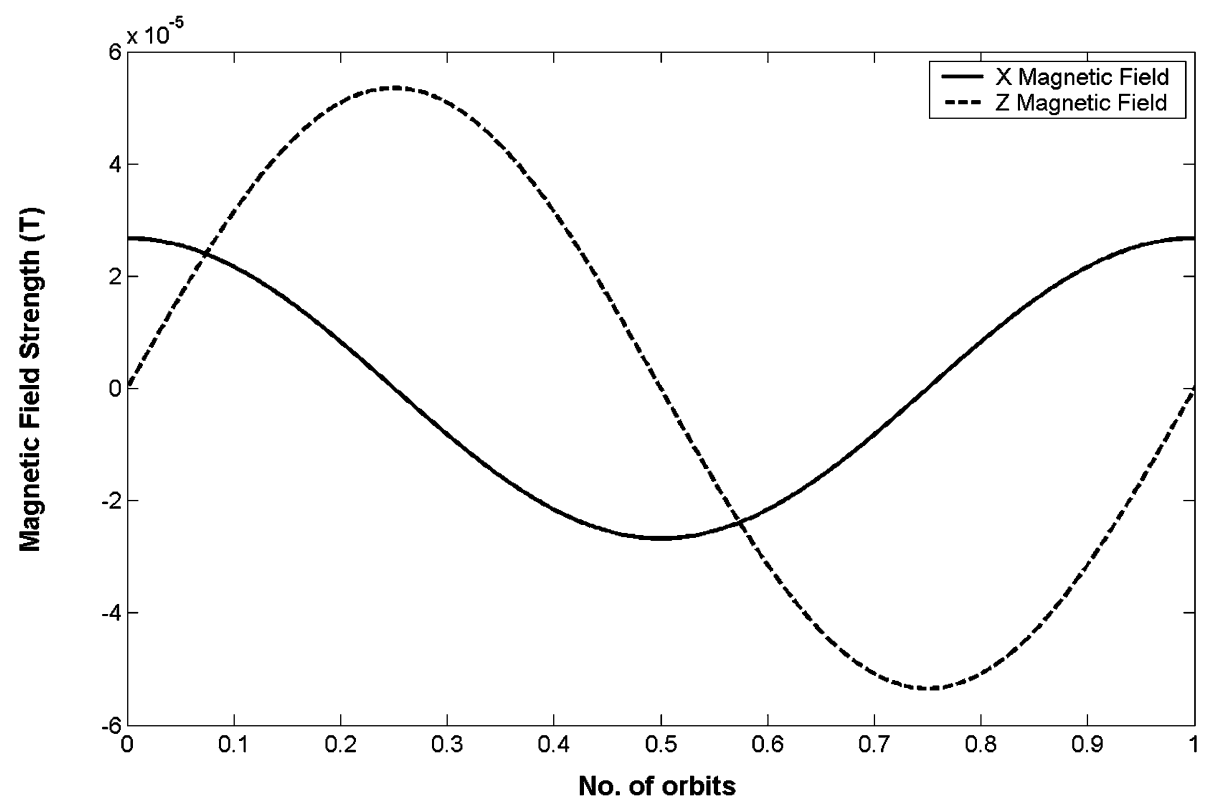

Fig. 1 Magnetic field in spacecraft co-ordinates

will never both equal zero and hence controllability of the pitch axis is always achievable through either the $M_{x}$ or $M_{z}$ dipole moment. As a result both of these dipoles are used specifically for control of the pitch axis. This only leaves the $M_{y}$ dipole to regulate both the roll and yaw axes; however, this single dipole control has already been shown to be feasible when considering control of a momentum-biased satellite [14-16].

With the pitch and lateral dynamics effectively being treated separately, equation (1) is decoupled to obtain the following relationship for the pitch dynamics

$$
\left[\begin{array}{c}
\dot{\theta} \\
\dot{\omega}_{y}
\end{array}\right]=\left[\begin{array}{cc}
0 & 1 \\
3 \omega_{0}^{2} \sigma_{2} & 0
\end{array}\right]\left[\begin{array}{c}
\theta \\
\omega_{y}
\end{array}\right]+\left[\begin{array}{c}
0 \\
1 / I_{y}
\end{array}\right] T_{y}
$$

With both the $M_{x}$ and $M_{z}$ dipoles available to control the pitch dynamics, a simple PD controller is implemented as

$$
T_{y}=-k_{\mathrm{p}} \theta-k_{\mathrm{d}} \omega_{y}
$$

where $k_{\mathrm{p}}$ and $k_{\mathrm{d}}$ are controller gains.

The controller gains are chosen to ensure the closed-loop eigenvalues have negative real parts, and hence asymptotic stability is guaranteed. Although this provides a required torque, this must be converted to equivalent dipole moments to be implemented. To define an explicit method of carrying this out, the lateral dynamics are reformulated.

\subsection{Reformulation of lateral dynamics}

From equation (1) the lateral dynamics can be defined by equation (8)

$$
\begin{aligned}
& {\left[\begin{array}{c}
\dot{\phi} \\
\dot{\psi} \\
\dot{\omega}_{x} \\
\dot{\omega}_{z}
\end{array}\right]=} \\
& {\left[\begin{array}{cccc}
0 & 0 & 1 & 0 \\
0 & 0 & 0 & 1 \\
-4 \omega_{0}^{2} \sigma_{1} & 0 & 0 & \omega_{0}\left(1-\sigma_{1}\right) \\
0 & \omega_{0}^{2} \sigma_{3} & -\omega_{0}\left(1+\sigma_{3}\right) & 0
\end{array}\right]\left[\begin{array}{c}
\phi \\
\psi \\
\omega_{x} \\
\omega_{z}
\end{array}\right]} \\
& +\left[\begin{array}{cc}
0 & 0 \\
0 & 0 \\
1 / I_{x} & 0 \\
0 & 1 / I_{z}
\end{array}\right]\left[\begin{array}{c}
T_{x} \\
T_{z}
\end{array}\right]
\end{aligned}
$$


From equation (2), representing the actuator dynamics, the control torques can be represented in terms of magnetic dipole moments in equations (9) and (10)

$$
\begin{aligned}
& {\left[\begin{array}{l}
T_{x} \\
T_{z}
\end{array}\right]=\left[\begin{array}{c}
B_{\mathrm{m}_{z}} \\
-B_{\mathrm{m}_{x}}
\end{array}\right] M_{y}+B_{\mathrm{m}_{y}}\left[\begin{array}{c}
-M_{z} \\
M_{x}
\end{array}\right]} \\
& -B_{\mathrm{m}_{z}} M_{x}+B_{\mathrm{m}_{x}} M_{z}=T_{y}
\end{aligned}
$$

As a design choice it is also possible to define the dot product of the dipole vector and magnetic field vector equal to zero [9]. Practically this minimizes the norm of the dipole vector and avoids applying dipole moments in the direction of the magnetic field, which produces zero torque and only serves to waste energy

$$
B_{\mathrm{m}_{x}} M_{x}+B_{\mathrm{m}_{z}} M_{z}=-B_{\mathrm{m}_{y}} M_{y}
$$

Combining equations (10) and (11) leads to the following relationship

$$
\left[\begin{array}{cc}
-B_{\mathrm{m}_{x}} & -B_{\mathrm{m}_{z}} \\
-B_{\mathrm{m}_{z}} & B_{\mathrm{m}_{x}}
\end{array}\right]\left[\begin{array}{c}
-M_{z} \\
M_{x}
\end{array}\right]=\left[\begin{array}{c}
T_{y} \\
-B_{\mathrm{m}_{y}} M_{y}
\end{array}\right]
$$

This can then be simplified to derive the following relationship for the lateral dipole moments

$$
\begin{aligned}
& {\left[\begin{array}{c}
-M_{z} \\
M_{x}
\end{array}\right]=} \\
& \frac{1}{-\left(B_{\mathrm{m}_{x}}^{2}+B_{\mathrm{m}_{z}}^{2}\right)}\left[\begin{array}{cc}
B_{\mathrm{m}_{x}} & -B_{\mathrm{m}_{y}} B_{\mathrm{m}_{z}} \\
B_{\mathrm{m}_{z}} & B_{\mathrm{m}_{x}} B_{\mathrm{m}_{y}}
\end{array}\right]\left[\begin{array}{c}
T_{y} \\
M_{y}
\end{array}\right]
\end{aligned}
$$

It should be noted that to avoid singularity problems when calculating the lateral dipole moments, $\left(B_{\mathrm{m}_{x}}^{2}+B_{\mathrm{m}_{z}}^{2}\right) \neq 0$. The only time this condition does not hold is for a satellite operating on an equatorial orbit. As magnetically actuated satellites typically operate on near-polar orbit, this numerical issue is not of concern. Substitution of equation (13) into equation (9) leads to

$$
\begin{aligned}
& {\left[\begin{array}{c}
T_{x} \\
T_{z}
\end{array}\right]=\frac{1}{\left(B_{\mathrm{m}_{x}}^{2}+B_{\mathrm{m}_{z}}^{2}\right)} \times} \\
& {\left[\begin{array}{cc}
-B_{\mathrm{m}_{x}} B_{\mathrm{m}_{y}} & B_{\mathrm{m}_{z}}\left(B_{\mathrm{m}_{x}}^{2}+B_{\mathrm{m}_{y}}^{2}+B_{\mathrm{m}_{z}}^{2}\right) \\
-B_{\mathrm{m}_{y}} B_{\mathrm{m}_{z}} & -B_{\mathrm{m}_{x}}\left(B_{\mathrm{m}_{x}}^{2}+B_{\mathrm{m}_{y}}^{2}+B_{\mathrm{m}_{z}}^{2}\right)
\end{array}\right]\left[\begin{array}{c}
T_{y} \\
M_{y}
\end{array}\right]}
\end{aligned}
$$

$$
\begin{aligned}
& {\left[\begin{array}{c}
\dot{\phi} \\
\dot{\psi} \\
\dot{\omega}_{x} \\
\dot{\omega}_{z}
\end{array}\right]=\left[\begin{array}{cccc}
0 & 0 & 1 & 0 \\
0 & 0 & 0 & 1 \\
-4 \omega_{0}^{2} \sigma_{1} & 0 & 0 & \omega_{0}\left(1-\sigma_{1}\right) \\
0 & \omega_{0}^{2} \sigma_{3} & -\omega_{0}\left(1+\sigma_{3}\right) & 0
\end{array}\right]} \\
& \times\left[\begin{array}{c}
\phi \\
\psi \\
\omega_{x} \\
\omega_{z}
\end{array}\right]+\frac{1}{\left(B_{\mathrm{m}_{x}}^{2}+B_{\mathrm{m}_{z}}^{2}\right)} \times \\
& {\left[\begin{array}{cc}
0 & 0 \\
0 & 0 \\
-B_{\mathrm{m}_{x}} B_{\mathrm{m}_{y}} / I_{x} & B_{\mathrm{m}_{z}}\left(B_{\mathrm{m}_{x}}^{2}+B_{\mathrm{m}_{y}}^{2}+B_{\mathrm{m}_{z}}^{2}\right) / I_{x} \\
-B_{\mathrm{m}_{y}} B_{\mathrm{m}_{z}} / I_{z} & -B_{\mathrm{m}_{x}}\left(B_{\mathrm{m}_{x}}^{2}+B_{\mathrm{m}_{y}}^{2}+B_{\mathrm{m}_{z}}^{2}\right) / I_{z}
\end{array}\right]\left[\begin{array}{c}
T_{y} \\
M_{y}
\end{array}\right]}
\end{aligned}
$$

For the purposes of the lateral controller design, $T_{y}$ may be treated as an external disturbance, with $M_{y}$ being the only control variable. As a result, equation (15) is written in the more compact form

$$
\dot{\boldsymbol{x}}_{\text {lat }}=\mathbf{A}_{\mathrm{lat}} \boldsymbol{x}_{\mathrm{lat}}+\mathbf{B}_{\mathrm{lat}} \boldsymbol{M}_{\mathrm{y}}
$$

where

$$
\begin{aligned}
& \mathbf{A}_{\mathrm{lat}}=\left[\begin{array}{cccc}
0 & 0 & 1 & 0 \\
0 & 0 & 0 & 1 \\
-4 \omega_{0}^{2} \sigma_{1} & 0 & 0 & \omega_{0}\left(1-\sigma_{1}\right) \\
0 & \omega_{0}^{2} \sigma_{3} & -\omega_{0}\left(1+\sigma_{3}\right) & 0
\end{array}\right] \\
& \mathbf{B}_{\mathrm{lat}}=\Delta\left[\begin{array}{c}
0 \\
0 \\
B_{\mathrm{m}_{z}} / I_{x} \\
-B_{\mathrm{m}_{x}} / I_{z}
\end{array}\right], \quad \Delta=\frac{\left(B_{\mathrm{m}_{x}}^{2}+B_{\mathrm{m}_{y}}^{2}+B_{\mathrm{m}_{z}}^{2}\right)}{\left(B_{\mathrm{m}_{x}}^{2}+B_{\mathrm{m}_{z}}^{2}\right)} \\
& \boldsymbol{x}_{\text {lat }}=\left[\begin{array}{llll}
\phi & \psi & \omega_{x} & \omega_{z}
\end{array}\right]^{\boldsymbol{T}}
\end{aligned}
$$

Remark 1. The reformulation of the dynamics now offers an explicit link between the pitch and lateral controllers. Once the pitch torque $T_{y}$ has been determined by the pitch controller and $M_{y}$ by the lateral controller, the full dipole vector can be determined using equation (13).

Remark 2. The neglecting of the pitch torque $T_{y}$ for the purpose of the lateral controller design is a reasonable assumption to make for two main 
reasons. First, the disturbance from the pitch control is attributable to the $B_{\mathrm{m}_{y}}$ magnetic field component, and in the spacecraft co-ordinate frame this is comparatively small in relation to the $B_{\mathrm{m}_{x}}$ and $B_{\mathrm{m}_{z}}$ components. Second, as the longitudinal dynamics are fully controllable, the closed-loop pitch dynamics are considerably faster than the lateral dynamics; therefore $\left\lfloor\theta \omega_{y}\right\rfloor \rightarrow 0$ and $T_{y} \rightarrow 0$ relatively quickly. This assumption is analytically supported through Lemma 2 in section 4.4.

\subsection{Stability enforced model predictive control of lateral dynamics}

The system in equation (16) is considered under a model predictive control scheme. This system is discretized to provide the following discrete-time model

$$
\boldsymbol{x}_{\text {lat }}(\boldsymbol{k}+1)=\boldsymbol{\Phi}_{\text {lat }} \boldsymbol{x}_{\text {lat }}(\boldsymbol{k})+\boldsymbol{\Gamma}_{\text {lat }}(\boldsymbol{k}) M_{y}(k)
$$

where

$$
\boldsymbol{\Phi}_{\text {lat }}=\exp \left(\mathbf{A}_{\text {lat }} T_{\mathrm{s}}\right), \quad \boldsymbol{\Gamma}_{\text {lat }}=\left[\int_{0}^{T_{\mathrm{s}}} \exp \left(\mathbf{A}_{\text {lat }} \eta\right) \mathrm{d} \eta\right] \mathbf{B}_{\text {lat }}
$$

and $T_{\mathrm{s}}$ is the controller sample time. This is directly analogous to equation (3) and can be iterated according to equation (4) to provide predictions of the system response. The optimal control signal is then calculated through minimization of the following performance index

$$
\begin{aligned}
J(k)= & \sum_{i=1}^{N} \hat{\boldsymbol{x}}_{\text {lat }}(k+i)^{T} \mathbf{Q} \hat{\boldsymbol{x}}_{\text {lat }}(k+i) \\
& \quad+\hat{M}_{y}(k+i-1)^{T} \mathbf{R} \hat{M}_{y}(k+i-1) \\
& +\hat{\boldsymbol{x}}_{\text {lat }}(k+N)^{T}(n \mathbf{Q}) \hat{\boldsymbol{x}}_{\text {lat }}(k+N)
\end{aligned}
$$

subject to

$$
\hat{\boldsymbol{x}}_{\text {lat }}(k+1)^{\boldsymbol{T}} \mathbf{P} \hat{\boldsymbol{x}}_{\text {lat }}(k+1) \leqslant \boldsymbol{x}_{\text {lat }}(k)^{\boldsymbol{T}} \mathbf{P} \boldsymbol{x}_{\text {lat }}(k)-\delta(k)
$$

where $\mathbf{Q}>0$ is the state weighting matrix, $\mathbf{R}>0$ is the control weighting matrix, $N$ is the prediction horizon, $n$ is a positive integer, $\delta(k) \geqslant 0$, and $\mathbf{P}>0$.

The constraint in equation (19) is included to enforce stability of the control law as described in section 3.3. As already discussed, it is imperative the constraint matrix $\mathbf{P}$ is chosen such that the optimization problem remains feasible. The problem of choosing an appropriate matrix becomes particularly challenging when considering time-varying systems.

In this paper the problem of choosing a feasible constraint matrix is tackled by first analysing the stability of an auxiliary controller. If it can be shown that a quadratic Lyapunov function exists under another control strategy, this Lyapunov function could be used as the constraint in equation (19), guaranteeing that the predictive controller would always have the option of implementing the auxiliary control law to satisfy the constraint. The auxiliary controller investigated is a simple velocity feedback scheme.

Lemma 1 (Krasovskii-LaSalle) [19]. Suppose there exists a function $V: \Re_{+} \times \Re^{n} \rightarrow \Re$ having the same period as the system such that $V$ is a positive definite function and is radially unbounded, and $\dot{V} \leqslant 0$. Define $R=\left\{\boldsymbol{x} \in \Re^{n}: \exists \boldsymbol{t} \geqslant 0\right.$ such that $\left.\dot{V}(t, x)=0\right\}$ and suppose $R$ does not contain any trajectories of the system other than the trivial trajectory. Then the equilibrium 0 is asymptotically stable.

Theorem 1. The system described by equation (16) is asymptotically stable under the velocity feedback $M_{y}=-K_{v}\left(B_{\mathrm{m}_{z}} \omega_{x}-B_{\mathrm{m}_{x}} \omega_{z}\right)$ for $K_{v}>0$.

Proof. Consider the Lyapunov function

$$
V=\boldsymbol{x}_{\text {lat }}^{\boldsymbol{T}} \mathbf{P}_{2} \boldsymbol{x}_{\text {lat }}
$$

where

$$
\mathbf{P}_{2}=\left[\begin{array}{cccc}
4 \omega_{0}^{2} \sigma_{1}\left(I_{x} / I_{z}\right) & 0 & 0 & 0 \\
0 & -\omega_{0}^{2} \sigma_{3} & 0 & 0 \\
0 & 0 & I_{x} / I_{z} & 0 \\
0 & 0 & 0 & 1
\end{array}\right]
$$

$V>0$ as for a satellite with GOCE configuration $\sigma_{1}>0$ and $\sigma_{3}<0$.

The time derivative of equation (20) is now shown to be negative semi-definite

$$
\begin{aligned}
\dot{V}= & \dot{\boldsymbol{x}}_{\text {lat }}^{T} \mathbf{P}_{2} \dot{\boldsymbol{x}}_{\text {lat }}+x_{\text {lat }}^{T} \mathbf{P}_{2} \dot{\boldsymbol{x}}_{\text {lat }} \\
\dot{V}= & \boldsymbol{x}_{\text {lat }}^{T}\left(\mathbf{A}_{\text {lat }}+\mathbf{B}_{\text {lat }} \mathbf{K}\right)^{T} \mathbf{P}_{2} \boldsymbol{x}_{\text {lat }} \\
& +\boldsymbol{x}_{\text {lat }}^{T} \mathbf{P}_{2}\left(\mathbf{A}_{\text {lat }}+\mathbf{B}_{\text {lat }} \mathbf{K}\right) \boldsymbol{x}_{\text {lat }}
\end{aligned}
$$

where

$$
\mathbf{K}=K_{\nu}\left\lfloor\begin{array}{llll}
0 & 0 & -B_{\mathrm{m}_{z}} & B_{\mathrm{m}_{x}}
\end{array}\right.
$$

Equation (22) can be written as 


$$
\dot{V}=\boldsymbol{x}_{\text {lat }}^{\boldsymbol{T}}\left(\mathbf{P}_{2}\left(\mathbf{A}_{\text {lat }}+\mathbf{B}_{\text {lat }} \mathbf{K}\right)+\left(\mathbf{P}_{2}\left(\mathbf{A}_{\text {lat }}+\mathbf{B}_{\text {lat }} \mathbf{K}\right)\right)^{\mathbf{T}}\right) \boldsymbol{x}_{\text {lat }}
$$

where, from equations (16) and (20)

$$
\begin{aligned}
& \mathbf{P}_{2}\left(\mathbf{A}_{\text {lat }}+\mathbf{B}_{\text {lat }} \mathbf{K}\right)= \\
& {\left[\begin{array}{cccc}
0 & 0 & 4 \omega_{0}^{2} \sigma_{1} \frac{I_{x}}{I_{z}} & 0 \\
0 & 0 & 0 & -\omega_{0}^{2} \sigma_{3} \\
-4 \omega_{0}^{2} \sigma_{1} \frac{I_{x}}{I_{L_{z}}} & 0 & -K_{\mathrm{v}} \Delta B_{\mathrm{m}_{z}}^{2} / I_{z} & \omega_{0}\left(1+\sigma_{3}\right)+\frac{K_{\mathrm{v}} \Delta B_{\mathrm{m}_{\mathrm{z}}} B_{\mathrm{m}_{z}}}{I_{z}} \\
0 & \omega_{0}^{2} \sigma_{3} & -\omega_{0}\left(1+\sigma_{3}\right)+\frac{K_{\mathrm{v}} \Delta B_{\mathrm{m}_{z}} B_{\mathrm{m}_{z}}}{I_{z}} & -K_{\mathrm{v}} \Delta B_{\mathrm{m}_{x}}^{2} / I_{z}
\end{array}\right]}
\end{aligned}
$$

Simplifying equation (23) allows the Lyapunov function to be written in its final form

$$
\dot{V}=-\frac{2 \Delta K_{\mathrm{v}}}{I_{z}}\left(B_{\mathrm{m}_{z}} \omega_{x}-B_{\mathrm{m}_{x}} \omega_{z}\right)^{2}
$$

Hence $\dot{V} \leqslant 0$ if $K_{\mathrm{v}}>0$.

It has been shown that the derivative of the Lyapunov function candidate is less than or equal to zero, which is a sufficient condition to establish stability of the system. As the system is periodic, asymptotic stability may be proven through Lemma 1.

It has already been shown that there exists a positive definite; function whose derivative is negative semi-definite; hence it need only be shown that there exist no trajectories of the system in $R$. It can be seen from equation (24) that $\dot{V}(t, x)=0$ when the following condition occurs

$$
\frac{\omega_{z}}{\omega_{x}}(t)=\frac{B_{\mathrm{m}_{z}}}{B_{\mathrm{m}_{x}}}(t)
$$

The condition in equation (25) coincides with a control input of $M_{y}=0$, and as a result it is quite simple to prove that $R$ does not contain any trajectories of the open-loop dynamics. The Earth's magnetic field is periodic with a time period equal to that of the Earth's rotation $\left(T_{\mathrm{e}}=24 \mathrm{~h}\right)$. Consider some initial time $t$, such that $B_{\mathrm{m}_{z}}(t)=0$. In order for equation (25) to hold, $\omega_{z}(t)=0$. As the Earth's magnetic field is periodic, $B_{\mathrm{m}_{z}}\left(t+n T_{\mathrm{e}}\right)=0$, where $n$ is an integer. The open-loop dynamics have approximate period of $T_{\text {open }}=5.6 T_{0}$ (where $T_{0}=2 \pi / \omega_{0}$ is the orbital period). As the GOCE satellite completes precisely 16 orbits per day, $T_{\text {earth }}=2.86 T_{\text {open }}$. If equation (25) is a trajectory of the system, $\omega_{z}\left(t+n 2.86 T_{\text {open }}\right)=0$, which clearly in general it will not. The relationship in equation (25) does not represent a trajectory of the system and hence according to Lemma 1 it is concluded that the system is asymptotically stable under the proposed control action.

It has now been shown that there always exists a control action $M_{y}=-K_{\mathrm{v}}\left(B_{\mathrm{m}_{z}} \omega_{x}-B_{\mathrm{m}_{x}} \omega_{z}\right)$ such that the Lyapunov function $V=\boldsymbol{x}_{\text {lat }}^{\boldsymbol{T}} \mathbf{P}_{2} \boldsymbol{x}_{\text {lat }}$ has negative semi-definite derivative. This directly implies that if an equivalent discrete-time Lyapunov function is defined as $V(k)=\boldsymbol{x}_{\text {lat }}(k)^{\boldsymbol{T}} \mathbf{P}_{2} \boldsymbol{x}_{\text {lat }}(k)$, there exists a control such that $V(k+1) \leqslant V(k)$. If the stability constraint in equation (19) is now chosen such that $\mathbf{P}=\mathbf{P}_{2}$, and $\delta(k)$ is chosen to be less than or equal to the reduction in the Lyapunov function achievable under the velocity controller, the stability constraint will always remain feasible. As it has now been shown that the stability constraint is always feasible, the system in equation (16) is locally asymptotically stable under the stability-enforced predictive controller according to the argument in section 3.3.

\subsection{Three-axis stability}

The analysis in the preceding sections is now combined to demonstrate asymptotic stability about all three axes.

Lemma 2 [20]. Consider the general system

$$
\begin{aligned}
& \dot{x}=f(x, z) \\
& \dot{z}=g(z)
\end{aligned}
$$

Suppose the equilibrium $x=0$ of $\dot{x}=f(x, 0)$ is locally asymptotically stable, and the equilibrium $z=0$ of $\dot{z}=g(z)$ is locally asymptotically stable. Then the equilibrium $(x, z)=(0,0)$ of the above system is locally asymptotically stable.

Theorem 2. The system in equation (1) is asymptotically stable with the origin under the combination of the PD control of the pitch dynamics in reference [7] and the model predictive control of the lateral dynamics proposed in section 4.3.

Proof. The full lateral dynamics in equation (15) can be written in the general form $\dot{x}_{\text {lat }}=f\left(x_{\text {lat }}, x_{\text {long }}\right)$. Through the analysis in section 4.3 the lateral dynamics are shown to be asymptotically stable under the proposed stability-enforced MPC algorithm, if the disturbance caused by the pitch axis control is neglected. As the disturbance from the pitch control is only zero when the state vector reaches zero, section 4.3 demonstrates that $x_{\text {lat }}=0$ of $\dot{x}_{\text {lat }}=f\left(x_{\text {lat }}, 0\right)$ is locally asymptotically stable.

The pitch dynamics in equation (6) under the control law in equation (7) can be written in the 
general form $\dot{x}_{\text {long }}=g\left(x_{\text {long }}\right)$. Through the analysis in section 4.1 the equilibrium $x_{\text {long }}=0$ of $\dot{x}_{\text {long }}=g\left(x_{\text {long }}\right)$ is shown to be locally asymptotically stable.

Through Lemma 2, if the system in equation (1) is subject to the proposed control action, the equilibrium $\left(x_{\text {lat }}, x_{\text {long }}\right)=(0,0)$ is locally asymptotically stable.

Remark 3. The proposed controller has now been shown to be asymptotically stable. Stability is guaranteed without the assumption that the Earth's magnetic field is periodic at the orbital frequency. Guaranteeing stability in this manner also provides robustness to any orbital drift or magnetic field modelling errors as, providing the current magnetic field can be accurately measured, the stability argument remains valid.

\section{SIMULATION}

To validate the analysis presented in section 4 and to demonstrate the performance achievable through the proposed controller, a simulation study is presented. The controller tuning parameters are summarized in Table 3. The controller sampling time is chosen as $10 \mathrm{~s}$ which, owing to the slow nature of the attitude dynamics, provides sufficiently high sampling (a $0.1 \mathrm{~Hz}$ sampling rate corresponds to 70 times the bandwidth frequency of the unstable pitch dynamics). Use of a longer sampling time also provides additional time to perform the necessary online computations for the predictive controller. Simulations have shown that long prediction and control horizons do not offer performance benefits for this constrained optimization problem and therefore a prediction and control horizon of five time steps gives the best performance at minimum computational burden.

In order to evaluate the performance of the proposed controller, the response is first assessed to initial conditions of $1^{\circ}$ pointing about each axis and angular rates of $0.0005 \mathrm{deg} / \mathrm{s}$ (typical for this phase of the attitude control). Figure 2 shows that the initial conditions are effectively removed while also demonstrating the stability of the controller. The allocation of two dipoles for specific control of the pitch axis easily handles the unstable dynamics. Although only controlled by a single dipole moment the roll/yaw dynamics are effectively handled, with the numerical simulations validating the Lyapunov function derived in the previous section.

As well as demonstrating good nominal performance, the controller must successfully regulate the satellite attitude when subjected to realistic disturbances due to the external environment. Owing to the low Earth orbit of the GOCE satellite, the attitude is significantly disturbed by aerodynamic torques from the upper atmosphere. To avoid decay of the satellite orbit, GOCE is also equipped with a thruster assembly which further disturbs the satellite attitude. Figures 3 to 6 demonstrate the ability of the controller to regulate the satellite attitude and meet the performance specifications, even in the presence of these disturbances. The attitude is regulated within $3^{\circ}$ of the nadir with the angular rate and accelerations remaining comfortably within required bounds. The magnetic dipole history is shown in Fig. 6 and shows the actuators operating well within the saturation limits of $400 \mathrm{Am}^{2}$.

The downside of a stability enforced approach is the computation imposed. The addition of a stability constraint means that the optimization problem cannot be solved by quadratic programming, which increases the computational burden. As already discussed, good performance can be achieved through a very short prediction horizon of just five time steps, and this limits much of the computational burden that would normally be imposed by much lengthier prediction horizons.

Table 3 Controller tuning parameters

\begin{tabular}{|c|c|c|c|c|c|}
\hline Controller & Tuning parameter & Value & & & \\
\hline Pitch PD control & $\begin{array}{l}\text { Proportional gain }\left(k_{\mathrm{p}}\right) \\
\text { Derivative gain }\left(k_{\mathrm{d}}\right)\end{array}$ & $\begin{array}{l}0.275 \\
37.7\end{array}$ & & & \\
\hline Lateral MPC & Prediction horizon $(N)$ & 5 & & & \\
\hline & Control horizon & 5 & & & \\
\hline & Sampling interval & $10 \mathrm{~s}$ & & & \\
\hline & Control weighting (R) & $1 e-5$ & & & \\
\hline & Terminal penalty $(n)$ & 500 & & & \\
\hline & State weighting matrix $(Q)$ & {$[570$} & 0 & 0 & 0 \\
\hline & & 0 & 1.3 & 0 & 0 \\
\hline & & 0 & 0 & $1 \mathrm{e} 9$ & 0 \\
\hline & & 0 & 0 & 0 & $1 \mathrm{e} 10$ \\
\hline
\end{tabular}



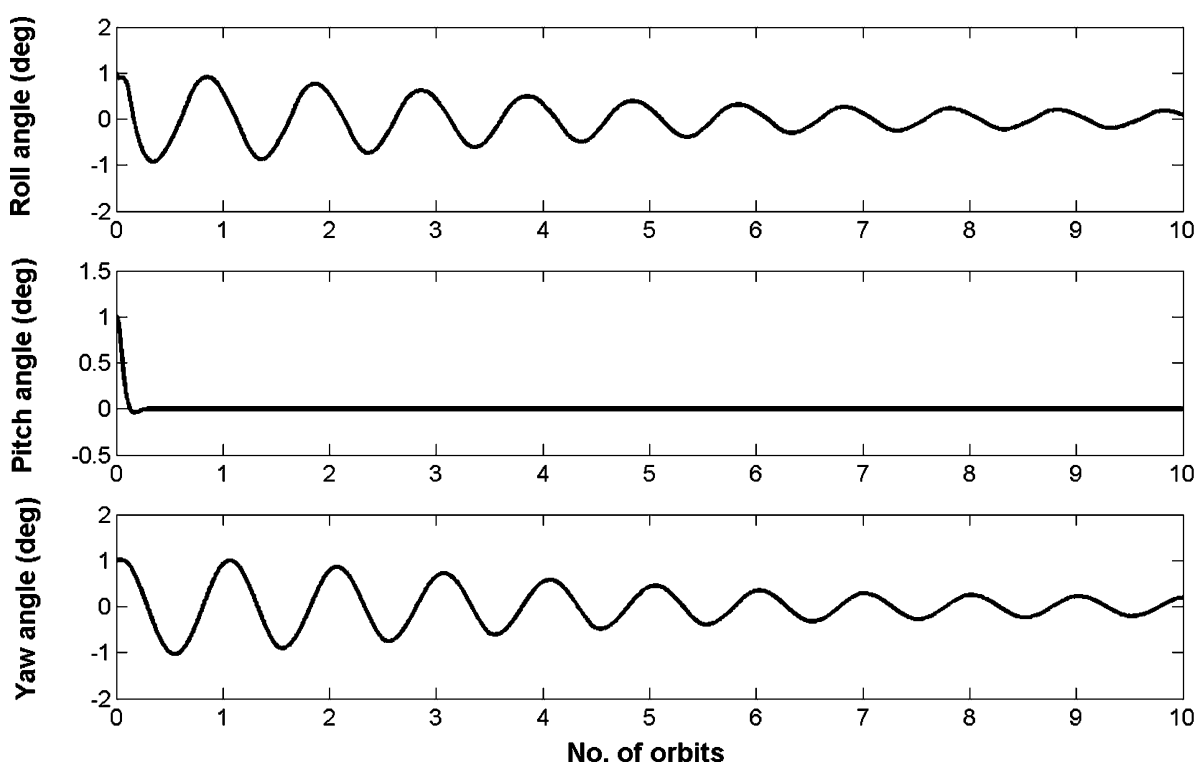

Fig. 2 Response of controller to initial conditions
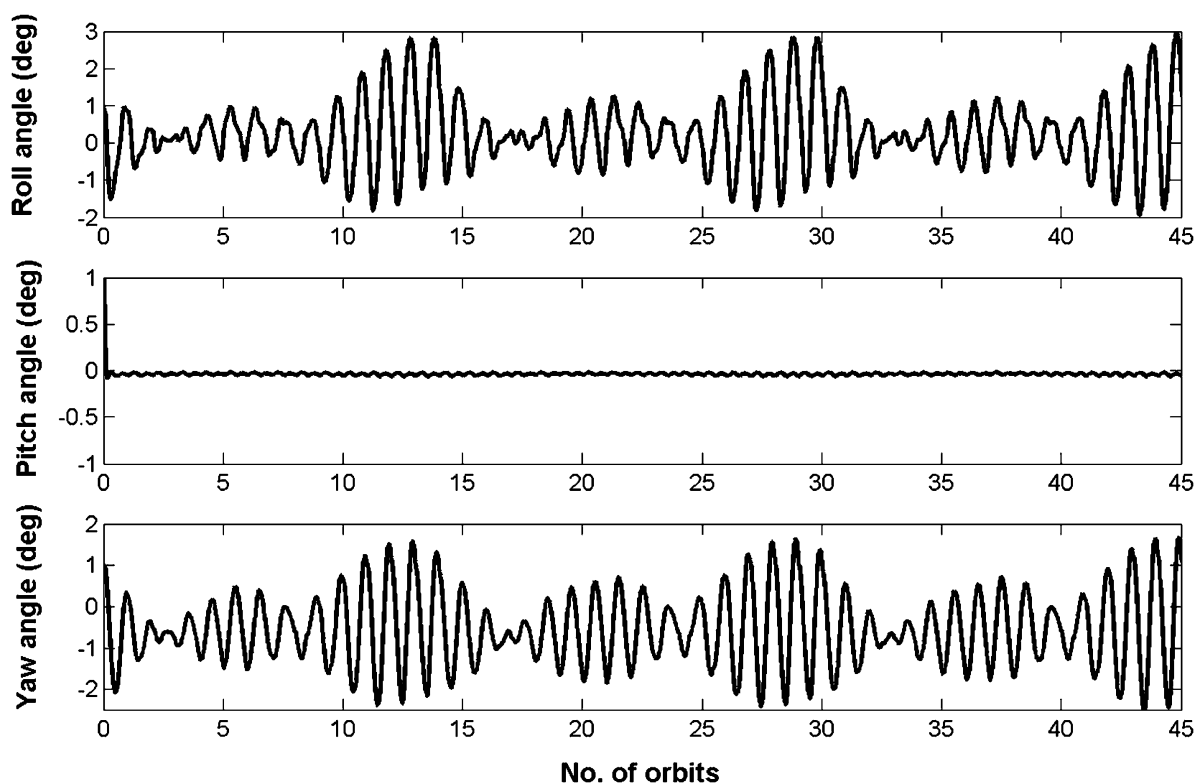

Fig. 3 Pointing time history subject to environmental disturbances

\section{CONCLUSION}

This paper has presented an asymptotically stable control law to regulate the attitude of a magnetically actuated spacecraft. A PD controller has been implemented on the pitch dynamics, while a stability constrained model predictive controller has been implemented for regulation of the lateral dynamics. A simulation study has investigated performance of the controller when applied to the GOCE satellite. Application of the stability enforced predictive controller verifies stability of the control scheme, presenting good performance even under significant environmental disturbance.

By adopting a constraint on the first state within the prediction horizon, the stability of the controller does not depend on the assumption that the geomagnetic field is periodic along the considered orbit. Use of such a constraint also guarantees stability in the presence of magnetic field modelling errors, as the current magnetic field can be accurately measured by onboard magnetometers.

Use of a stability enforced approach does lead to an increase in computational burden owing to loss 


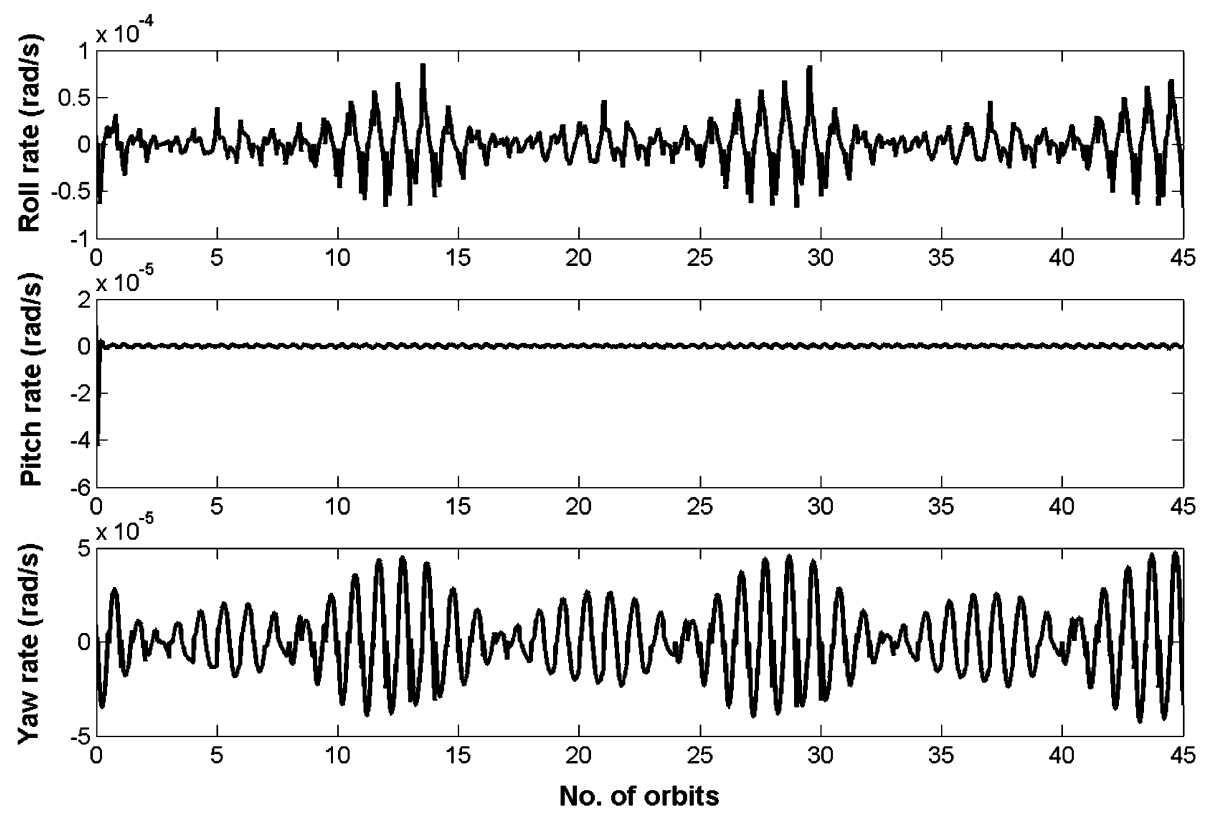

Fig. 4 Angular rate time history subject to environmental disturbances
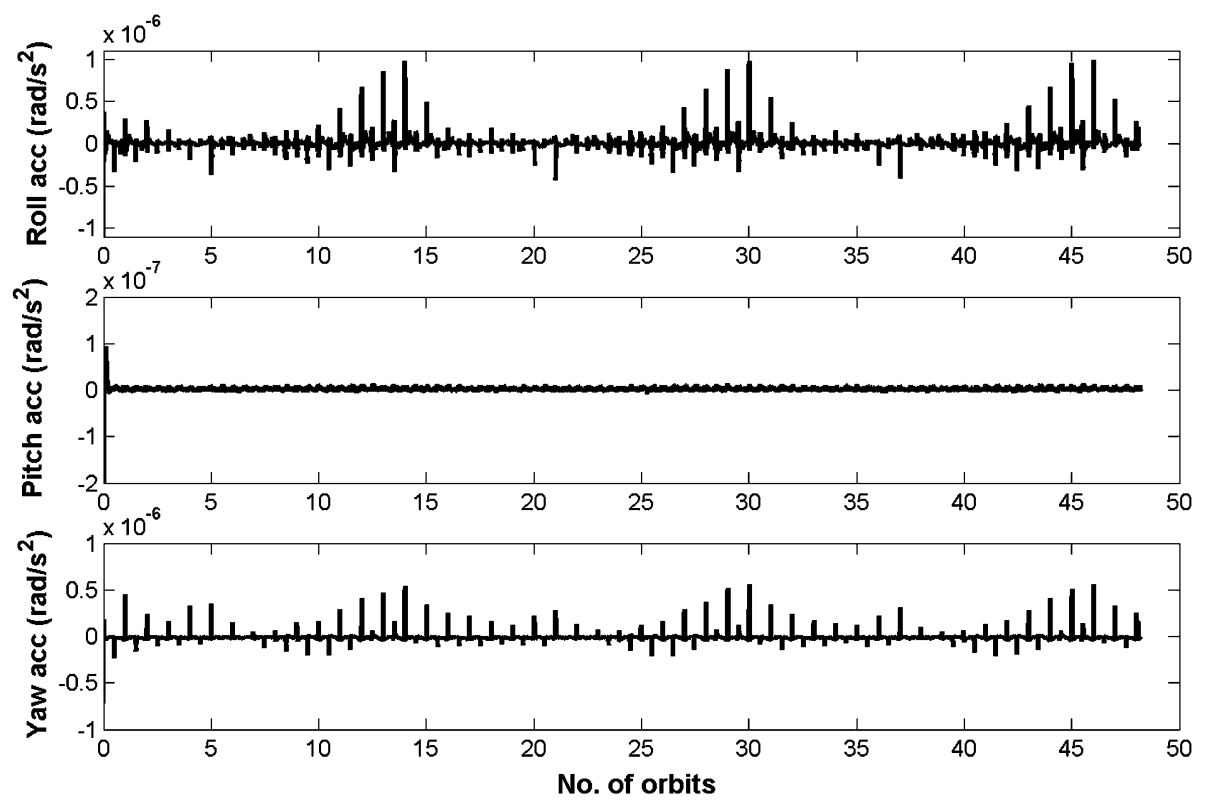

Fig. 5 Angular acceleration time history subject to environmental disturbances

of a quadratic programming optimization problem. Results show, however, that good performance can be achieved with very short prediction horizons, which minimizes the computational burden imposed by the constraint.

\section{ACKNOWLEDGEMENTS}

The authors would like to thank Denis Fertin and Christian Philippe for their useful comments and provision of the GOCE simulator. They would also like to thank ESA for financial support for this research.

\section{REFERENCES}

1 Martel, F., Pal, P., and Psiaki, M. Active magnetic control system for gravity gradient stabilized spacecraft. In 2nd Annular AIAA/USU Conference on Small Satellites, Logan, Utah, USA, 1988. 

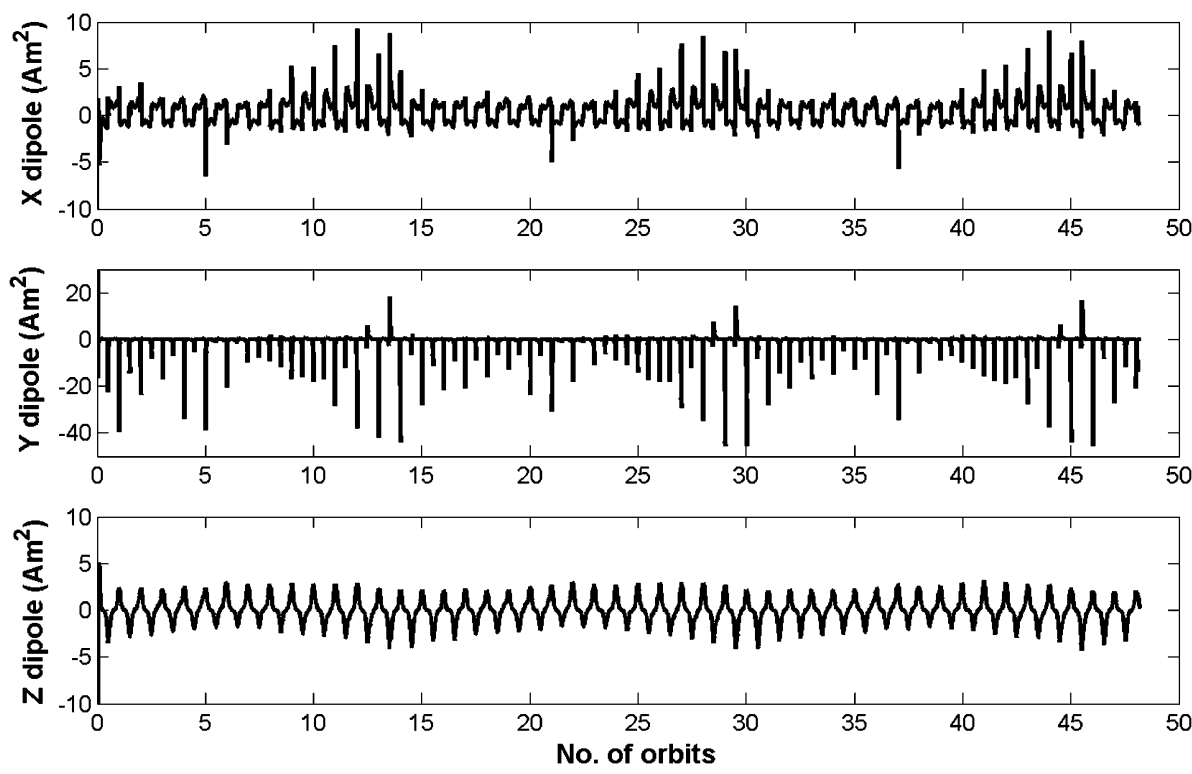

Fig. 6 Magnetic dipole time history subject to environmental disturbances

2 Arduini, C. and Baiocco, P. Active magnetic damping attitude control for gravity gradient stabilized spacecraft. J. Guidance Control, 1997, 20(1), 117-122.

3 Sechi, G., André, G., Andreis, D., and Saponara, M. Magnetic attitude control of the GOCE satellite. Proceedings of the 6th International ESA Conference on Guidance, Navigation and Control Systems, Loutraki, Greece, 2006.

4 Psiaki, M. Magnetic torquer attitude control via asymptotic periodic linear quadratic regulation. AIAA Guidance, Navigation, and Control Conference, Denver, Colorado, USA, 2000.

5 Lovera, M., De Marchi, E., and Bittanti, S. Periodic attitude control techniques for small satellite with magnetic actuators. IEEE Trans. Control Systems Technol., 2002, 10(1), 90-95.

6 Lovera, M. and Astolfi, A. Global magnetic attitude control of spacecraft in the presence of gravity gradient. Proceedings of 42nd IEEE Conference on Decision and Control, Maui, Hawaii, USA, 2003.

7 Lovera, M. and Astolfi, A. Spacecraft attitude control using magnetic actuators. Automatica, 2004, 40, 1405-1414.

8 Wisniewski, R. Satellite attitude control using only electromagnetic actuation. PhD Thesis, Aalborg University, Denmark, 1996.

9 Silani, E. and Lovera, M. Magnetic spacecraft attitude control: a survey and some new results. Control Engng Practice, 2005, 13, 357-371.

10 Krogstad, T., Gravdahl, J., and Tondel, P. Explicit model predictive control of a satellite with magnetic torquers. Proceedings of the 2005 IEEE International Symposium on Control and Automation, Limassol, Cyprus, 2005.
11 Wisniewski, R. and Blanke, M. Fully magnetic attitude control for spacecraft subject to gravity gradient. Automatica, 1999, 35, 1201-1214.

12 Wood, M., Chen, W.-H., and Fertin, D. Model predictive control of low Earth orbiting spacecraft with magneto-torquers. Proceedings of IEEE International Conference on Control Applications, Munich, 2006.

13 Martella, P., Sechi, G., and Canuto, E. The GOCE drag free and attitude control design aspects and expected performance. Proceedings of the 5th International ESA Conference on Spacecraft Guidance, Navigation and Control Systems, Frascati, Italy, 2002.

14 Stickler, A. C. and Alfriend, K. T. Elementary magnetic attitude control system. J. Spacecraft Rockets, 1976, 13(5), 282-287.

15 Pittelkau, M. Optimal periodic control for spacecraft pointing and attitude determination. J. Guidance, Control Dynamics, 1993, 16(6), 1078-1084.

16 Varga, A. and Pieters, S. Gradient-based approach to solve optimal periodic output feedback control problems. Automatica, 1998, 34, 477-481.

17 Lee, J. W., Kwon, W. H., and Choi, J. On stability of constrained receding horizon control with finite terminal weighting matrix. Automatica, 1998, 34(12), 1607-1612.

18 Kothare, S. and Morari, M. Contractive model predictive control for constrained non-linear systems. IEEE Trans Automatic Control, 2000, 45(6), 1053-1071.

19 Vidyasagar, M. Nonlinear systems analysis, 1978 (Prentice Hall, New York).

20 Isidori, A. Nonlinear control systems II, 1999 (Springer Verlag, London). 


\section{APPENDIX}

\section{Notation}

A, $\mathbf{A}_{\text {lat }}$

B, $\mathbf{B}_{\text {lat }}$

$\boldsymbol{B}_{\mathrm{m}}$

I

$J$

K

$k_{\mathrm{p}}, k_{\mathrm{d}}$

$K_{\mathrm{v}}$

$\boldsymbol{M}$

$n$

$\mathbf{P}$ continuous-time system matrix

for full and lateral satellite

dynamics

continuous-time control matrix for

full and lateral satellite dynamics

Earth's magnetic field vector in

spacecraft co-ordinates

satellite inertia matrix

cost function

feedback gain for stable velocity

controller

proportional and derivative gains for pitch controller

velocity feedback gain

magnetic dipole vector

terminal penalty

Lyapunov function matrix
$T_{\mathrm{e}}$

$T_{0}$

$\boldsymbol{U}$

V

$\boldsymbol{x}$

$X$

$\Gamma$

$\sigma_{1}, \sigma_{2}, \sigma_{3}$

$\varphi, \theta, \psi$

$\Phi$

$\omega_{x}, \omega_{y}, \omega_{z}$

$\omega_{0}$ state weighting matrix

control weighting matrix

control torque

Earth's rotational period

orbital period

predicted future control sequence

Lyapunov function

state vector

predicted state vector

discrete-time control matrix for general time-varying system

inertia ratios

pointing angle of spacecraft about

local-level co-ordinate frame

discrete-time system matrix for gen-

eral time-varying system

angular rate of spacecraft about

local-level co-ordinate frame

orbital rate 